\title{
Spin Relaxation in $s$-Wave Superconductors in the Presence of Resonant Spin-Flip Scatterers
}

\author{
Denis Kochan $\odot, *$ Michael Barth, Andreas Costa, Klaus Richter, and Jaroslav Fabian \\ Institute for Theoretical Physics, University of Regensburg, 93040 Regensburg, Germany
}

(Received 14 February 2019; revised 22 May 2020; accepted 22 July 2020; published 18 August 2020)

\begin{abstract}
Employing analytical methods and quantum transport simulations we investigate the relaxation of quasiparticle spins in graphene proximitized by an $s$-wave superconductor in the presence of resonant magnetic and spin-orbit active impurities. Off resonance, the relaxation increases with decreasing temperature when electrons scatter off magnetic impurities-the Hebel-Slichter effect-and decreases when impurities have spin-orbit coupling. This distinct temperature dependence (not present in the normal state) uniquely discriminates between the two scattering mechanisms. However, we show that the HebelSlichter picture breaks down at resonances. The emergence of Yu-Shiba-Rusinov bound states within the superconducting gap redistributes the spectral weight away from magnetic resonances. The result is opposite to the Hebel-Slichter expectation: the spin relaxation decreases with decreasing temperature. Our findings hold for generic $s$-wave superconductors with resonant magnetic impurities, but also, as we show, for resonant magnetic Josephson junctions.
\end{abstract}

DOI: 10.1103/PhysRevLett.125.087001

Introduction.-Superconducting spintronics investigates the interplay between the electron spin phenomena [1] and macroscopic quantum coherence of superconducting structures [2-4]. A versatile platform for superconducting spintronics is offered by 2D layered materials. Indeed, there is a growing family of 2D superconductors-twisted bilayer graphene [5,6], 2D topological insulators [7,8], or transition-metal dichalcogenides [9-13]—which could serve as a source of Cooper pairs. At the same time there are high-mobility 2D (semi)metals and semiconductors whose spin properties, in particular spin relaxation (SR), can profoundly change when proximitized by superconductors.

Measurements of SR in graphene have not yielded a unique mechanism for electron spin flips [14-23]. Ensuing intense scientific discussions [24-30] have focused on spinorbit and exchange impurities as possible culprits. The principal difficulty in setting one against the other lies, unlike in conventional materials [1], in the absence of a systematic temperature behavior of the measured spin relaxation. However, the absence of SR anisotropy [21] points towards magnetic resonant impurities $[31,32]$ as the main source of spin-flip scattering in graphene.

Here we show that in (proximitized) superconducting graphene (SCG) the two types of impurities yield distinct temperature characteristics due to coherence effects. Particularly striking is the prediction that resonant magnetic scatterers cause SR whose temperature dependence is opposite to that predicted by the perturbative HebelSlichter effect [33-35]. Since the nonperturbative analytic and quantum transport simulation methods we use are not specific to graphene, this prediction applies to resonant scattering in all $s$-wave superconductors. Furthermore, we demonstrate that it also applies to superconducting resonant Josephson junctions with magnetic tunnel barriers.

Although measurements of SR in SCG have not yet been performed (which makes theoretical predictions particularly motivating), they are within the current experimental reach. Indeed, proximity induced superconductivity in graphene has been experimentally demonstrated in lateral Josephson junctions [36-39] and vertical stack geometries [40,41], as well as in alkaline-intercalated graphite [42-44]. The induced superconducting gap ranges from tens of $\mu \mathrm{eV}$ [36] to $1 \mathrm{meV}$ [45] ( $\left.T_{c} \simeq 6.593 \mathrm{~K}\right)$. Both $s$-wave [36] and $p$-wave [41] pairings were convincingly demonstrated; see the comprehensive review Ref. [46] for more details.

Rationale.-Yafet showed [47], using the first order perturbation theory, that the SR rate in superconductors is modified from the normal state as $1 / \tau_{s}^{\mathrm{SC}} \sim$ $\left\langle\left(u_{\mathbf{k}} u_{\mathbf{q}} \pm v_{\mathbf{k}} v_{\mathbf{q}}\right)^{2} / \tau_{s}^{\mathrm{N}}\right\rangle$, where $u$ and $v$ are standard BCS coherence factors and $\langle\cdots\rangle$ denotes thermal broadening. The plus sign applies to interactions that are odd under time reversal, such as exchange. As a result, $1 / \tau_{s}^{\mathrm{SC}}>1 / \tau_{s}^{\mathrm{N}}$, which is the Hebel-Slichter effect [33-35]. On the other hand, the minus sign is for time-reversal symmetric interactions such as spin-orbit coupling, in which case $1 / \tau_{s}^{\mathrm{SC}}<1 / \tau_{s}^{\mathrm{N}}$, as demonstrated experimentally for aluminum $[48,49][50]$. We will see that in SCG $1 / \tau_{s}^{\mathrm{N}}$ and $1 / \tau_{s}^{\mathrm{SC}}$ can differ by orders of magnitude due to the coherence effects, which allows for an unprecedented experimental feasibility to disentangle the dominant SR mechanism. Our methodology overcomes two shortcomings of the standard 
theory of Yafet, Hebel, and Slichter. First, we calculate the SR rate to all orders of perturbation theory allowing us to consider resonant scattering and, second, we also include subgap Yu-Shiba-Rusinov (YSR) states [54,66-68] which have no normal-state counterpart and which take away considerable spectral weight from the scattering states.

Model and methodology.-To describe SCG we use the tight-binding Hamiltonian [69],

$$
\begin{aligned}
H_{g}= & -\sum_{m, n, \sigma}\left(t \delta_{\langle m n\rangle}+\mu \delta_{m n}\right) c_{m \sigma}^{\dagger} c_{n \sigma} \\
& +\Delta \sum_{m} c_{m \uparrow}^{\dagger} c_{m \downarrow}^{\dagger}+\text { H.c.; }
\end{aligned}
$$

$t=2.6 \mathrm{eV}$ stands for the conventional nearest neighbor hopping, $\mu$ for the chemical potential (doping level) taking the normal phase's charge neutrality point as a reference, and $\Delta$ models the $T$-dependent global on-site $s$-wave pairing. We assume the BCS-like temperature dependence $\Delta(T)=\Delta_{0} \tanh \left[1.74 \sqrt{T_{c} / T-1}\right]$ with realistic proximity values of $\Delta_{0}=1 \mathrm{meV}$ and $T_{c} \simeq 6.593 \mathrm{~K}$. The operator $c_{m \sigma}^{(\dagger)}$ annihilates (creates) an electron with spin $\sigma$ at the graphene lattice site $m, \delta_{m n}$ represents the usual Kronecker symbol, and $\delta_{\langle m n\rangle}$ its nearest-neighbor analog (unity for the direct graphene nearest-neighbor sites and zero otherwise). Orbital interactions of carbon at site $m=0$ with an adatom - annihilation and creation operators $d_{\sigma}$ and $d_{\sigma}^{\dagger}$-are governed by the impurity-site hybridization $\omega$, on-site energy $\varepsilon$, and the proximity pairing $\Delta$, combining into [70] (see the inset of Fig. 1)

$V_{o}=\sum_{\sigma}\left[(\varepsilon-\mu) d_{\sigma}^{\dagger} d_{\sigma}+\omega d_{\sigma}^{\dagger} c_{0 \sigma}\right]+\Delta d_{\uparrow}^{\dagger} d_{\downarrow}^{\dagger}+$ H.c..

This orbital perturbation is complemented by a local spindependent term $V_{s}$ comprising (1) exchange interaction, $V_{s}^{(1)}=-J \mathbf{S} \cdot \mathbf{S}$, between the itinerant electron spin $\mathbf{s}$ and the impurity 1/2-spin $\mathbf{S}$ [31], and (2) local spin orbit coupling (SOC) in the vicinity of an adatom [50,55$57,71,72]$. To be specific, we use hydrogen and fluorine adatoms, both of which induce sizable SOC enhancements $[55,56]$ and can also carry magnetic moments [73-80]. We also assume low concentrations $\eta$ (per carbon atom) of dilute spin-active impurities [81] for the independent scatter picture to be valid.

Our methodology employs the full analytical approach, calculating the spin-flip rates from the T matrix, as well as numerical KWANT simulations of spin-flip scattering probabilities, providing together coherent and consistent qualitative and quantitative pictures. The detailed methodology is in the Supplemental Material [50].

Results.-Adatoms on graphene give rise to resonances [82-85], which strongly modify graphene transport properties [85-91], particularly when they lie close to the Dirac point. Figure 1 demonstrates how normal-state resonances affect the population of quasiparticle states in SCG at

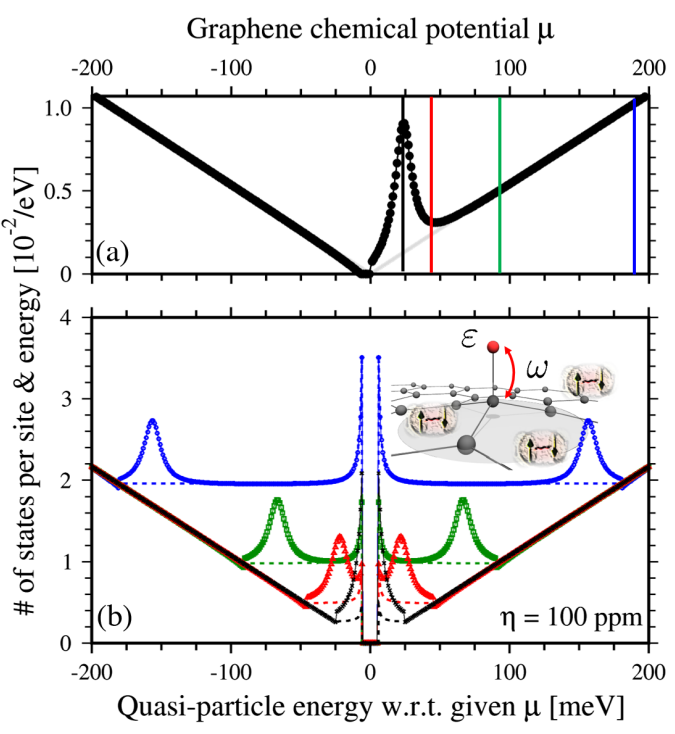

FIG. 1. Calculated density of states. (a) Graphene's normalstate DOS (black dots) as a function of the chemical potential $\mu$ for $100 \mathrm{ppm}$ of resonant nonmagnetic impurities. A pronounced resonant peak emerges at $\mu=24 \mathrm{meV}$; the gray line is for pristine graphene. Black, red, green, and blue vertical lines represent chemical potentials at which superconductivity is turned on with gap $\Delta_{0}=5 \mathrm{meV}$. The corresponding quasiparticle (QP) DOS is shown in (b). Black symbols stand for $\mu=24 \mathrm{meV}$, red triangles for $\mu=45 \mathrm{meV}$, green squares for $\mu=90 \mathrm{meV}$, and blue circles for $\mu=180 \mathrm{meV}$. Dashed lines with the same colors serve as guides for the eye and display the corresponding QPDOS in clean SCG. Resonant enhancements near the coherence peaks appear for chemical potentials close to the normal-phase resonances. Inset: Tight-binding description of an adatom absorbed on SCG. For DOS we used hybridization $\omega=5.5 \mathrm{eV}$ and on-site energy $\varepsilon=0.26 \mathrm{eV}$.

different chemical potentials. Panel 1(a) shows the DOS of graphene covered by $100 \mathrm{ppm}$ of resonant nonmagnetic impurities, while panel 1(b) displays the corresponding quasiparticle DOS (QPDOS). We present resonant and offresonant doping limits and conclude that the QPDOS gets strongly modified near the coherence peaks as $\mu$ approaches the normal-state resonance. This makes sense since for given $\mu$ BCS theory gives QPDOS $(E)=$ $\left[E /\left(\sqrt{E^{2}-\Delta^{2}}\right)\right] \operatorname{DOS}(\mu)$.

Figure 2 illustrates various characteristics of spin-flip scattering off magnetic impurities in normal and superconducting graphene for two representative impurities: hydrogen-panels 2(a), 2(c), 2(e)-and fluorine-panels 2(b), 2(d), 2(f). Particularly, Figs. 2(a) and 2(b) display the SR rates in SCG for different temperatures in the presence of $280 \mathrm{ppm}$ of magnetic impurities, as a function of the chemical potential $\mu$ and the superconducting gap with temperature $T$. Solid lines corresponds to analytical T-matrix calculation, and the symbols with the same color to the corresponding KWANT simulation, for details see Ref. [50]. Hydrogen [31] with a nonzero magnetic moment $-\omega=7.5 \mathrm{eV}, \varepsilon=0.16 \mathrm{eV}$, and $J=-0.4 \mathrm{eV}$-gives rise 

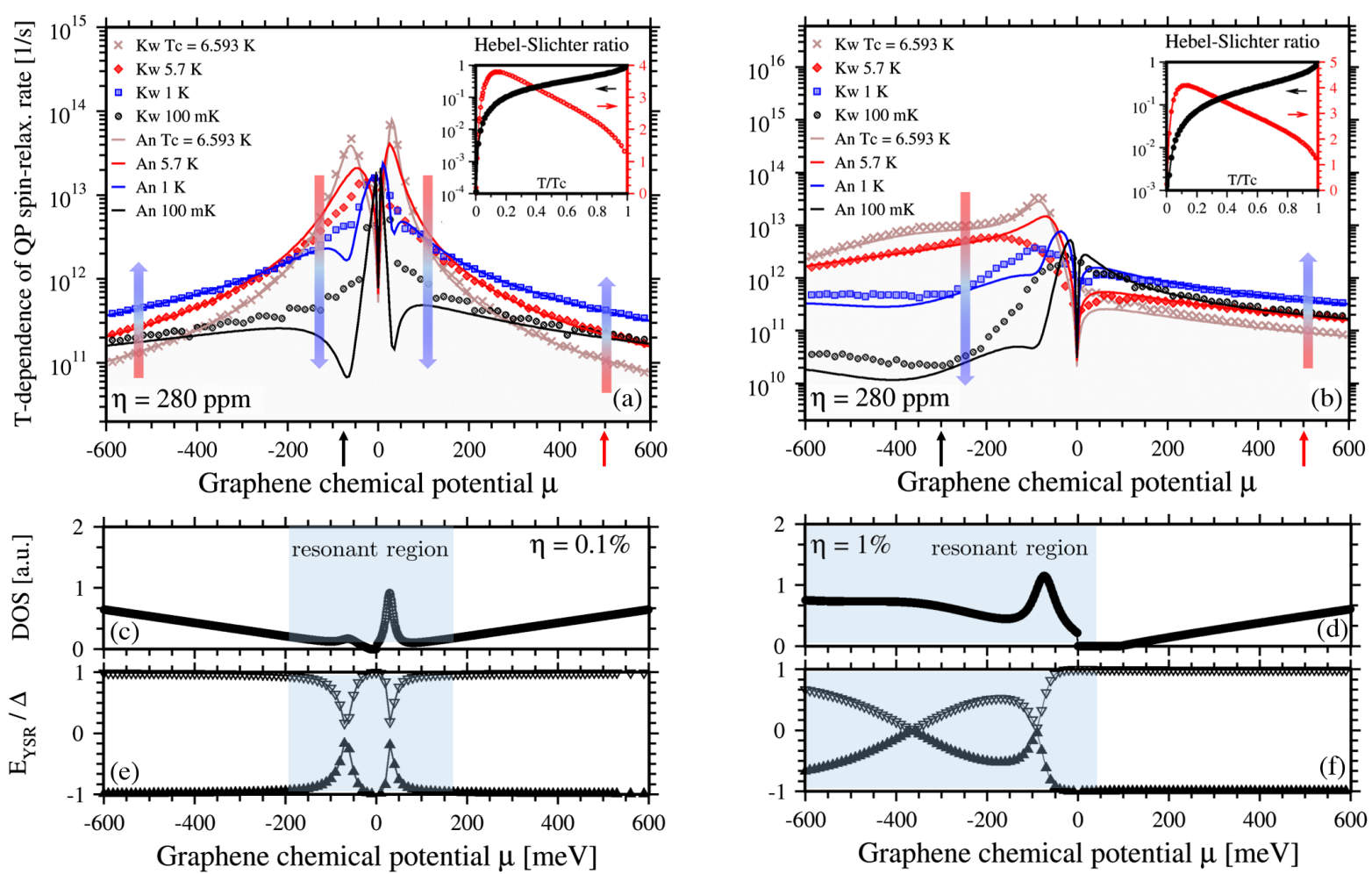

FIG. 2. Quasiparticle SR rates in SCG (as functions of $\mu$ ) at different temperatures (different colors) for 280 ppm of (a) hydrogen and (b) fluorine magnetic impurities, solid lines-analytical calculations, symbols-KwANT simulations. The SR rates increase off resonance whereas they decrease in the resonances. Rainbow arrows (coded in colors of temperature descent) indicate the SR rates' increasing or decreasing trends with lowered $T$ compared to the normal phase. The insets show the corresponding Hebel-Slichter ratios, $\left(1 / \tau_{s}^{\mathrm{SC}}\right) /\left(1 / \tau_{s}^{\mathrm{N}}\right)$, as functions of $T / T_{c}$ and at two representative Fermi energies (indicated by black and red arrow ticks on the horizontal axis): resonant ( $\mu=-80 \mathrm{meV}$ for hydrogen and $\mu=-300 \mathrm{meV}$ for fluorine; see black circled data with values at left logarithmic axis) and off resonant ( $\mu=500 \mathrm{meV}$ for both cases; see red circled data with values at right linear axis). KwANT simulation used a scattering region with $W=299 a$ and $L=5 a$, a small misalignment around charge neutrality originates from finite-size effects. Panels (c) and (d) show the normal-phase DOS obtained from analytical calculation in the presence of magnetic moments, and resonant (shaded) and off-resonant (white) doping regions; to increase the visibility, the impurity concentrations were exaggerated. Panels (e) and (f) obtained form the poles of the analytical Green functions display the subgap YSR states' energies for hydrogen and fluorine (as functions of $\mu$ ). Smaller SR rates in (a) and (b) are correlated with the normal-phase resonances in (c) and (d), and the YSR states in (e) and (f) with energies deep inside the gap.

to a narrow normal-state resonant region near the Dirac point; see the corresponding magnetic DOS in Fig. 2(c) (concentration $\eta=0.1 \%$ is exaggerated for resolution purposes). In contrast, fluorine- $\omega=5.5 \mathrm{eV}$, $\varepsilon=-2.2 \mathrm{eV}$, and $J=0.5 \mathrm{eV}$-develops [56,79] a wide resonance region spreading below the Dirac point; see the magnetic DOS in Fig. 2(d) (again with the elevated concentration $\eta=1 \%$ ).

The striking impact of resonances on quasiparticle SR rates is seen from Figs. 2(a) and 2(b). The shaded regions show the SR rate in the normal phase $\left(T=T_{c}\right)$. Lowering $T$ below superconducting $T_{c}$ we observe an intriguing behavior: for off-resonant doping regions, $1 / \tau_{s}^{\mathrm{SC}}>1 / \tau_{s}^{\mathrm{N}}$ in accordance with the Hebel-Slichter scenario [33-35], whereas quasiparticle SR sharply drops, $1 / \tau_{s}^{\mathrm{SC}} \ll 1 / \tau_{s}^{\mathrm{N}}$, at resonances. To further quantify those effects, the insets of Figs. 2(a) and 2(b) represent the corresponding Hebel-Slichter ratios, $\left(1 / \tau_{s}^{\mathrm{SC}}\right) /\left(1 / \tau_{s}^{\mathrm{N}}\right)$, taken at two representative $\mu$ 's as functions of $T / T_{c}$. For the offresonant value of $\mu=500 \mathrm{meV}$, both impurity cases lead to a notable enhancement of the superconducting SR rates by almost a factor of 4 (graphs with red symbols), while we see a strong decrease of the SR rates by almost 3 orders of magnitude (graphs with black symbols in logarithmic scale) inside the resonant regions-we use $\mu=-80 \mathrm{meV}$ for hydrogen and $\mu=-300 \mathrm{meV}$ for fluorine. This can serve as powerful experimental evidence-observing strongly depleted SR rates in the superconducting phase when lowering $T$ signifies the presence of resonant magnetic impurities.

To explain this peculiar resonant depletion of QP SRs in SCG, which is at odds with the well-understood resonant enhancement [31,32,92-95] in the normal phase, we calculate the energies (poles of $\mathrm{T}$ matrix, [96]) of the corresponding YSR states [66-68] which develop around magnetic impurities [97,98], see Figs. 2(e) and 2(f). We 
deduce that the YSR states lie deep inside the superconducting gap at doping levels that correspond to resonances in the normal phase of graphene. This offers an explanation why the SR rates of quasiparticles sharply decrease. Resonant scattering of QPs counts many contributions, especially those from multiple scatterings and those from virtual states' tunnelings. Schematically, the scattering matrix element can be written as $V_{\alpha \alpha}+V_{\alpha I}\left[(|I\rangle\langle I|) /\left(E_{\alpha}-E_{I}+i 0_{+}\right)\right] V_{I \alpha}+\cdots$, where $E_{I}$ represents the energy of any intermediate state (extended or subgap), and $E_{\alpha}$ stands for the energy of an incident QP state. The dominant elements $V_{\alpha I}$ for spin-flip processes arise from a strong overlap of quasiparticle states $\alpha$ with the magnetic impurity level $I=$ YSR since only the latter gives rise to QP spin flips. While the matrix elements $V_{\alpha \mathrm{YSR}}$ are roughly the same for all extended QP states $\alpha$ at the coherence peaks, independently of doping (impurity in our model acts like $\delta$ function), this is not true for the energy differences $E_{\alpha}-E_{\mathrm{YSR}}$ in the denominator. Those are, in fact, small in the off-resonant region since $E_{\mathrm{YSR}}$ are aligned with the superconducting gap edges and become large at the resonances, where $E_{\mathrm{YSR}}$ are deep inside the gap. That this must cause the reduced SR is clear from the $T$ dependence of the SR rates; for higher $T$, $\Delta$ gets smaller and hence also the difference $E_{\alpha}-E_{\mathrm{YSR}}$. The opposite conclusion would follow from Yafet's formula, which does not account for the formation of subgap bound states.

At very low temperatures-data for $T=100 \mathrm{mK}$ displayed by black solid lines (analytic) and black empty dots (numeric) in Figs. 2(a) and (b) - the SR rates drop down at low dopings since the related QPDOS, $\quad E /\left(\sqrt{E^{2}-\Delta^{2}}\right) \operatorname{DOS}(\mu)[-(\partial g / \partial E)]$, becomes substantially suppressed upon thermal smearing. However, at larger dopings this suppression is counteracted by the elevated $\operatorname{DOS}(\mu)$.

Finally, we turn to SR due to spin-orbit active impurities. Figure 3 displays the calculated SR rates in SCG as functions of doping at different temperatures for fluorine impurities, see Ref. [50] for the hydrogen which shows the same qualitative features. We find that the SR rates in SCG decrease (as predicted by Yafet [47]) by one order of magnitude with lowering $T$, giving sizable experimental signals. At resonances SR rates get enhanced, similarly to what was predicted for the normal state [58]. This is because a quasiparticle in a resonant state has sufficient time to experience SOC and flip its spin, what gives rise to the increased SR rates, although somewhat less than in the normal state due to lower group velocities of quasiparticles. Thus, observing a global decrease of the SR rate with lowered $T$ spanning a wide doping region would be an unprecedented experimental signal for SOC-dominated SR.

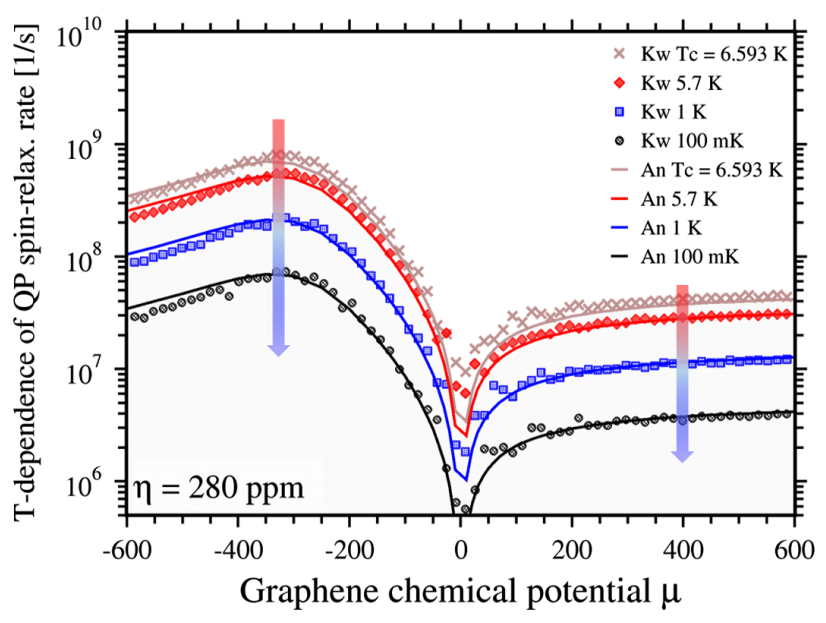

FIG. 3. SR rates in SCG (as functions of $\mu$ ) at different temperatures (different colors) due to locally enhanced SOC for $280 \mathrm{ppm}$ of fluorine impurities, solid lines-analytical calculations, symbols-KWANT simulations. The SR rates decrease almost uniformly with lowering temperature $T$; their decrease becomes steeper and would saturate as $T \rightarrow 0$. Similarly to the normal state, the SR rates are enhanced at resonances. Rainbow arrows (coded in colors of temperature descent) indicate the SR rates' decreasing trend with lowered $T$.

Although motivated by superconducting spintronics we used primarily SCG to perform detailed simulations, our findings are also qualitative and thus expected to be valid for resonant scattering off magnetic impurities in generic $s$ wave superconductors. In fact, we have analyzed a simple 1D model of a resonant Josephson junction with magnetic tunnel barriers [50], which explicitly demonstrates the reduction of spin-flip probabilities of quasiparticles at resonances due to the appearance of deep immersed YSR states.

Conclusions.-We have shown that the spin relaxation of quasiparticles due to resonant magnetic impurities has the opposite temperature dependence from what is predicted as the Hebel-Slichter effect. The reason is emergence of subgap YSR states which redistribute the spectral weight away from the resonances. The anomalous decrease of resonant SR can span 3 to 4 orders of magnitude, making it a robust and verifiable experimental tool.

The authors thank Dr. Ferenc Simon, Dr. Yuriy Pogorelov, Dr. Lucia Komendová, Dr. Cosimo Gorini, Dr. Marcin Kurpas, and Dr. Benedikt Scharf for useful discussions, and Dr. Jeongsu Lee for helpful tips regarding numerical implementation. The authors acknowledge support from Deutsche Forschungsgemeinschaft (DFG, German Research Foundation)—Project-ID 314695032SFB 1277 (Subprojects A07, A09, B07), the EU Seventh Framework Programme under Grant Agreement 
No. 604391 (Graphene Flagship), and the International Doctorate Program Topological Insulators of the Elite Network of Bavaria.

*denis.kochan@ur.de

[1] I. Žutić, J. Fabian, and S. D. Sarma, Rev. Mod. Phys. 76, 323 (2004).

[2] M. Eschrig, Phys. Today 64, No. 1, 43 (2011).

[3] M. Eschrig, Rep. Prog. Phys. 78, 104501 (2015).

[4] J. Linder and J. W. A. Robinson, Nat. Phys. 11, 307 (2015).

[5] Y. Cao, V. Fatemi, S. Fang, K. Watanabe, T. Taniguchi, E. Kaxiras, and P. Jarillo-Herrero, Nature (London) 556, 43 (2018).

[6] M. Yankowitz, S. Chen, H. Polshyn, Y. Zhang, K. Watanabe, T. Taniguchi, D. Graf, A. F. Young, and C. R. Dean, Science 363, 1059 (2019).

[7] E. Sajadi, T. Palomaki, Z. Fei, W. Zhao, P. Bement, C. Olsen, S. Luescher, X. Xu, J. A. Folk, and D. H. Cobden, Science 362, 922 (2018).

[8] V. Fatemi, S. Wu, Y. Cao, L. Bretheau, Q. D. Gibson, K. Watanabe, T. Taniguchi, R. J. Cava, and P. Jarillo-Herrero, Science 362, 926 (2018).

[9] W. Shi, J. Ye, Y. Zhang, R. Suzuki, M. Yoshida, J. Miyazaki, N. Inoue, Y. Saito, and Y. Iwasa, Sci. Rep. 5, 12534 (2015).

[10] S. Jo, D. Costanzo, H. Berger, and A. F. Morpurgo, Nano Lett. 15, 1197 (2015).

[11] X. Xi, Z. Wang, W. Zhao, J.-H. Park, K. T. Law, H. Berger, L. Forró, J. Shan, and K. F. Mak, Nat. Phys. 12, 139 (2016).

[12] E. Navarro-Moratalla, J. O. Island, S. Mañas-Valero, E. Pinilla-Cienfuegos, A. Castellanos-Gomez, J. Quereda, G. Rubio-Bollinger, L. Chirolli, J. A. Silva-Guillén, N. Agraït, G. A. Steele, F. Guinea, H. S. J. van der Zant, and E. Coronado, Nat. Commun. 7, 11043 (2016).

[13] D. Costanzo, S. Jo, H. Berger, and A. F. Morpurgo, Nat. Nanotechnol. 11, 339 (2016).

[14] N. Tombros, C. Jozsa, M. Popinciuc, H. T. Jonkman, and B. J. van Wees, Nature (London) 448, 571 (2007).

[15] M. Ohishi, M. Shiraishi, R. Nouchi, T. Nozaki, T. Shinjo, and Y. Suzuki, Jpn. J. Appl. Phys. 46, L605 (2007).

[16] K. Pi, W. Han, K. M. McCreary, A. G. Swartz, Y. Li, and R. K. Kawakami, Phys. Rev. Lett. 104, 187201 (2010).

[17] T. Y. Yang, J. Balakrishnan, F. Volmer, A. Avsar, M. Jaiswal, J. Samm, S. R. Ali, A. Pachoud, M. Zeng, M. Popinciuc, G. Güntherodt, B. Beschoten, and B. Özyilmaz, Phys. Rev. Lett. 107, 047206 (2011).

[18] A. Avsar, T.-Y. Yang, S. Bae, J. Balakrishnan, F. Volmer, M. Jaiswal, Z. Yi, S. R. Ali, G. Güntherodt, B. H. Hong, B. Beschoten, and B. Özyilmaz, Nano Lett. 11, 2363 (2011).

[19] M. B. Lundeberg, R. Yang, J. Renard, and J. A. Folk, Phys. Rev. Lett. 110, 156601 (2013).

[20] M. H. D. Guimarães, P. J. Zomer, J. Ingla-Aynés, J. C. Brant, N. Tombros, and B. J. van Wees, Phys. Rev. Lett. 113, 086602 (2014).

[21] B. Raes, J. E. Scheerder, M. V. Costache, F. Bonell, J. F. Sierra, J. Cuppens, J. Van de Vondel, and S. O. Valenzuela, Nat. Commun. 7, 11444 (2016).

[22] M. Drögeler, C. Franzen, F. Volmer, T. Pohlmann, L. Banszerus, M. Wolter, K. Watanabe, T. Taniguchi, C. Stampfer, and B. Beschoten, Nano Lett. 16, 3533 (2016).
[23] S. Dushenko, H. Ago, K. Kawahara, T. Tsuda, S. Kuwabata, T. Takenobu, T. Shinjo, Y. Ando, and M. Shiraishi, Phys. Rev. Lett. 116, 166102 (2016).

[24] P. Seneor, B. Dlubak, M.-B. Martin, A. Anane, H. Jaffres, and A. Fert, MRS Bull. 37, 1245 (2012).

[25] W. Han, R. K. Kawakami, M. Gmitra, and J. Fabian, Nat. Nanotechnol. 9, 794 (2014).

[26] S. Roche and S. O. Valenzuela, J. Phys. D 47, 094011 (2014).

[27] S. Roche, J. Åkerman, B. Beschoten, J.-C. Charlier, M. Chshiev, S. P. Dash, B. Dlubak, J. Fabian, A. Fert, M. Guimarães, F. Guinea, I. Grigorieva, C. Schönenberger, P. Seneor, C. Stampfer, S. O. Valenzuela, X. Waintal, and B. van Wees, 2D Mater. 2, 030202 (2015).

[28] Y. P. Feng, L. Shen, M. Yang, A. Wang, M. Zeng, Q. Wu, S. Chintalapati, and C.-R. Chang, Wiley Interdiscip. Rev. 7, e1313 (2017).

[29] J. H. Garcia, M. Vila, A. W. Cummings, and S. Roche, Chem. Soc. Rev. 47, 3359 (2018).

[30] A. G. Rybkin, A. A. Rybkina, M. M. Otrokov, O. Y. Vilkov, I. I. Klimovskikh, A. E. Petukhov, M. V. Filianina, V. Y. Voroshnin, I. P. Rusinov, A. Ernst, A. Arnau, E. V. Chulkov, and A. M. Shikin, Nano Lett. 18, 1564 (2018).

[31] D. Kochan, M. Gmitra, and J. Fabian, Phys. Rev. Lett. 112, 116602 (2014).

[32] D. Kochan, S. Irmer, M. Gmitra, and J. Fabian, Phys. Rev. Lett. 115, 196601 (2015).

[33] L. C. Hebel and C. P. Slichter, Phys. Rev. 107, 901 (1957).

[34] L. C. Hebel and C. P. Slichter, Phys. Rev. 113, 1504 (1959).

[35] L. C. Hebel, Phys. Rev. 116, 79 (1959).

[36] H. B. Heersche, P. Jarillo-Herrero, J. B. Oostinga, L. M. K. Vandersypen, and A. F. Morpurgo, Nature (London) 446, 56 (2007).

[37] K. Komatsu, C. Li, S. Autier-Laurent, H. Bouchiat, and S. Guéron, Phys. Rev. B 86, 115412 (2012).

[38] V. E. Calado, S. Goswami, G. Nanda, M. Diez, A. R. Akhmerov, K. Watanabe, T. Taniguchi, T. M. Klapwijk, and L. M. K. Vandersypen, Nat. Nanotechnol. 10, 761 (2015).

[39] D. I. Indolese, R. Delagrange, P. Makk, J. R. Wallbank, K. Wanatabe, T. Taniguchi, and C. Schönenberger, Phys. Rev. Lett. 121, 137701 (2018).

[40] C. Tonnoir, A. Kimouche, J. Coraux, L. Magaud, B. Delsol, B. Gilles, and C. Chapelier, Phys. Rev. Lett. 111, 246805 (2013).

[41] A. Di Bernardo, O. Millo, M. Barbone, H. Alpern, Y. Kalcheim, U. Sassi, A. K. Ott, D. De Fazio, D. Yoon, M. Amado, A. C. Ferrari, J. Linder, and J. W. A. Robinson, Nat. Commun. 8, 14024 (2017).

[42] K. Li, X. Feng, W. Zhang, Y. Ou, L. Chen, K. He, L.-L. Wang, L. Guo, G. Liu, Q.-K. Xue, and X. Ma, Appl. Phys. Lett. 103, 062601 (2013).

[43] B. M. Ludbrook, G. Levy, P. Nigge, M. Zonno, M. Schneider, D. J. Dvorak, C. N. Veenstra, S. Zhdanovich, D. Wong, P. Dosanjh, C. Straßer, A. Stöhr, S. Forti, C. R. Ast, U. Starke, and A. Damascelli, Proc. Natl. Acad. Sci. U.S.A. 112, 11795 (2015).

[44] J. Chapman, Y. Su, C. A. Howard, D. Kundys, A. N. Grigorenko, F. Guinea, A. K. Geim, I. V. Grigorieva, and R. R. Nair, Sci. Rep. 6, 23254 (2016). 
[45] P. Rickhaus, M. Weiss, L. Marot, and C. Schönenberger, Nano Lett. 12, 1942 (2012).

[46] G.-H. Lee and H.-J. Lee, Rep. Prog. Phys. 81, 056502 (2018).

[47] Y. Yafet, Phys. Lett. 98A, 287 (1983).

[48] H. Yang, S.-H. Yang, S. Takahashi, S. Maekawa, and S. S. P. Parkin, Nat. Mater. 9, 586 (2010).

[49] C. H. L. Quay, M. Weideneder, Y. Chiffaudel, C. Strunk, and M. Aprili, Nat. Commun. 6, 8660 (2015).

[50] See Supplemental Material at http://link.aps.org/ supplemental/10.1103/PhysRevLett.125.087001 for more details about the charge and spin accumulation of QPs in superconductors, their nonequilibrium separation, and relaxation, consult Refs. [51-53], including Refs. [1,31, 33-35,47,54-65].

[51] H. L. Zhao and S. Hershfield, Phys. Rev. B 52, 3632 (1995).

[52] T. Yamashita, S. Takahashi, H. Imamura, and S. Maekawa, Phys. Rev. B 65, 172509 (2002).

[53] C. H. L. Quay, D. Chevallier, C. Bena, and M. Aprili, Nat. Phys. 9, 84 (2013).

[54] A. Costa, J. Fabian, and D. Kochan, Phys. Rev. B 98, 134511 (2018).

[55] M. Gmitra, D. Kochan, and J. Fabian, Phys. Rev. Lett. 110, 246602 (2013).

[56] S. Irmer, T. Frank, S. Putz, M. Gmitra, D. Kochan, and J. Fabian, Phys. Rev. B 91, 115141 (2015).

[57] D. Kochan, S. Irmer, and J. Fabian, Phys. Rev. B 95, 165415 (2017).

[58] J. Bundesmann, D. Kochan, F. Tkatschenko, J. Fabian, and K. Richter, Phys. Rev. B 92, 081403(R) (2015).

[59] J. R. Schrieffer, Theory of Superconductivity (Benjamin, New York, 1964).

[60] M. Gmitra, S. Konschuh, C. Ertler, C. Ambrosch-Draxl, and J. Fabian, Phys. Rev. B 80, 235431 (2009).

[61] J. Fabian and M. W. Wu, in Handbook of Spin Transport and Magnetism, edited by E. Tsymbal and I. Žutić (CRC Press, Taylor \& Francis Group, Boca Raton, 2012), pp. 303-327.

[62] D. Kochan, M. Gmitra, and J. Fabian, in Symmetry, Spin Dynamics and the Properties of Nanostructures, Lecture Notes of the 11th International School on Theoretical Physics, edited by J. Barnaś, V. Dugaev, and A. Wal (World Scientific, Singapore, 2014), pp. 136-162.

[63] C. W. Groth, M. Wimmer, A. R. Akhmerov, and X. Waintal, New J. Phys. 16, 063065 (2014).

[64] P. G. De Gennes, Superconductivity of Metals and Alloys (Addison Wesley, Redwood City, 1989).

[65] A. Costa and J. Fabian, Phys. Rev. B 101, 104508 (2020).

[66] L. Yu, Acta Phys. Sin. 21, 75 (1965).

[67] H. Shiba, Prog. Theor. Phys. 40, 435 (1968).

[68] A. I. Rusinov, Zh. Eksp. Teor. Fiz. 9, 146 (1969); JETP Lett. 9, 85 (1969).

[69] B. Uchoa and A. H. C. Neto, Phys. Rev. Lett. 98, 146801 (2007).

[70] C. F. Ratto and A. Blandin, Phys. Rev. 156, 513 (1967).

[71] K. Zollner, T. Frank, S. Irmer, M. Gmitra, D. Kochan, and J. Fabian, Phys. Rev. B 93, 045423 (2016).

[72] T. Frank, S. Irmer, M. Gmitra, D. Kochan, and J. Fabian, Phys. Rev. B 95, 035402 (2017).
[73] O. V. Yazyev, Rep. Prog. Phys. 73, 056501 (2010).

[74] L. Xie, X. Wang, J. Lu, Z. Ni, Z. Luo, H. Mao, R. Wang, Y. Wang, H. Huang, D. Qi, R. Liu, T. Yu, Z. Shen, T. Wu, H. Peng, B. Özyilmaz, K. Loh, A. T. S. Wee, Ariando, and W. Chen, Appl. Phys. Lett. 98, 193113 (2011).

[75] X. Hong, K. Zou, B. Wang, S.-H. Cheng, and J. Zhu, Phys. Rev. Lett. 108, 226602 (2012).

[76] R. R. Nair, M. Sepioni, I.-L. Tsai, O. Lehtinen, J. Keinonen, A. V. Krasheninnikov, T. Thomson, A. K. Geim, and I. V. Grigorieva, Nat. Phys. 8, 199 (2012).

[77] H. Gonzalez-Herrero, J. M. Gomez-Rodriguez, P. Mallet, M. Moaied, J. J. Palacios, C. Salgado, M. M. Ugeda, J.-Y. Veuillen, F. Yndurain, and I. Brihuega, Science 352, 437 (2016).

[78] K. Szałowski, Carbon 108, 327 (2016).

[79] S. Wellnhofer, A. Stabile, D. Kochan, M. Gmitra, Y.-W. Chuang, J. Zhu, and J. Fabian, Phys. Rev. B 100, 035421 (2019).

[80] F. J. Sousa, B. Amorim, and E. V. Castro, arXiv:1901.08614.

[81] M. J. Zuckermann, Phys. Rev. 140, A899 (1965).

[82] Y. V. Skrypnyk and V. M. Loktev, Phys. Rev. B 75, 245401 (2007).

[83] T. O. Wehling, S. Yuan, A. I. Lichtenstein, A. K. Geim, and M. I. Katsnelson, Phys. Rev. Lett. 105, 056802 (2010).

[84] A. Ferreira and E. R. Mucciolo, Phys. Rev. Lett. 115, 106601 (2015).

[85] S. Irmer, D. Kochan, J. Lee, and J. Fabian, Phys. Rev. B 97, 075417 (2018).

[86] Y. V. Skrypnyk and V. M. Loktev, Phys. Rev. B 82, 085436 (2010).

[87] A. Ferreira, T. G. Rappoport, M. A. Cazalilla, and A. H. C. Neto, Phys. Rev. Lett. 112, 066601 (2014).

[88] A. A. Stabile, A. Ferreira, J. Li, N. M. R. Peres, and J. Zhu, Phys. Rev. B 92, 121411(R) (2015).

[89] J. H. Garcia and T. G. Rappoport, 2D Mater. 3, 024007 (2016).

[90] J. Katoch, T. Zhu, D. Kochan, S. Singh, J. Fabian, and R. K. Kawakami, Phys. Rev. Lett. 121, 136801 (2018).

[91] J. Lee, D. Kochan, and J. Fabian, Phys. Rev. B 99, 035412 (2019).

[92] D. Soriano, D. V. Tuan, S. M.-M. Dubois, M. Gmitra, A. W. Cummings, D. Kochan, F. Ortmann, J.-C. Charlier, J. Fabian, and S. Roche, 2D Mater. 2, 022002 (2015).

[93] J. Wilhelm, M. Walz, and F. Evers, Phys. Rev. B 92, 014405 (2015).

[94] V. Miranda, E. Mucciolo, and C. Lewenkopf, J. Phys. Chem. Solids 128, 169 (2019).

[95] M. R. Thomsen, M. M. Ervasti, A. Harju, and T.G. Pedersen, Phys. Rev. B 92, 195408 (2015).

[96] V. M. Loktev and Y. G. Pogorelov, Dopants and Impurities in High-Tc Superconductors (Akademperiodyka, Kyiv, 2015).

[97] T. O. Wehling, H. P. Dahal, A. I. Lichtenstein, and A. V. Balatsky, Phys. Rev. B 78, 035414 (2008).

[98] J. L. Lado and J. Fernández-Rossier, 2D Mater. 3, 025001 (2016). 